\title{
The DB gap of white dwarfs and semiconvection
}

\author{
Hiromoto Shibahashi \\ Department of Astronomy, University of Tokyo, Tokyo 113-0033, Japan \\ email: shibahashi@astron.s.u-tokyo.ac.jp
}

\begin{abstract}
A remarkable and intriguing fact is that few DB white dwarfs, i.e., objects with a helium-rich atmosphere, are found in the effective temperature range between 45000-30000 K, and this exclusion zone is known as the DB gap. Since the temperatures of the blue and the red edges of the DB gap coincide with the effective temperatures where HeII/III and HeI/II convection zones show up respectively, convective mixing is suspected to be the cause of the appearance of DBs outside the DB gap. Chemical separation due to gravitational settling in the convectively stable atmosphere is then suspected to be the cause of the presence of the DB gap. The white dwarfs which turn back to DBs after the DB gap phase are expected to have a semiconvective layer, which is superadiabatic but convectively stable, when they are located near the red edge of the DB gap. Such white dwarfs are expected to be pulsationally unstable. Discovery of pulsation in them will open up a new class of pulsating white dwarfs to asteroseismic study.
\end{abstract}

Keywords. Convection, diffusion, instabilities, stars: white dwarfs, stars: oscillations

\section{Introduction}

In terms of surface composition, white dwarfs come in two major subgroups: those showing hydrogen Balmer lines only, which constitute about $80 \%$ of all white dwarfs, and those showing helium lines only. The former are classified as DA white dwarfs, and the latter are termed DB or DO white dwarfs, depending on which types of helium lines are seen; neutral He lines (DBs) or ionized He lines (DOs).

It should be noted here that the classification of white dwarfs is not analogous to that of the main-sequence stars in the sense that it reflects compositions in their atmospheres and not the temperatures. The distribution of the subgroups of white dwarfs with respect to effective temperature has been carefully investigated, and it has been found that DA white dwarfs are seen in a wide temperature range, but, on the other hand, among the helium white dwarfs, DO white dwarfs are seen in the temperature range of $T_{\text {eff }}>$ $45000 \mathrm{~K}$ and DB white dwarfs are found for $T_{\text {eff }}<30000 \mathrm{~K}$. There are few white dwarfs showing helium lines between $T_{\text {eff }} \simeq 45000 \mathrm{~K}$ and $T_{\text {eff }} \simeq 30000 \mathrm{~K}$, and this exclusion zone is known as the "DB gap" (Liebert 1986; Liebert et al. 1986; Sion 1984). The existence of this gap presents a puzzle concerning the origin and evolution of helium atmosphere white dwarfs.

\section{Spectral evolution of white dwarfs}

A simple picture of parallel sequences of $\mathrm{H}$ and He-rich white dwarfs may not be acceptable as an explanation for the DB gap, since accelerated evolution of He-rich white dwarfs between $T_{\text {eff }} \simeq 45000 \mathrm{~K}$ and $T_{\text {eff }} \simeq 30000 \mathrm{~K}$ does not seem to make sense. Rather, the presence of the DB gap implies that white dwarfs may change their spectroscopic type as they cool down, probably due to the interplay of diffusion and convection, though the 
chemical evolution is quite complicated and not completely understood (Bergeron et al. 1997; Hansen \& Liebert 2003).

Fontaine \& Wesemael (1987) are the first who proposed a possible scenario along this line to explain the DB gap. They assumed that all white dwarfs have a common origin, planetary nebula nuclei, with some hydrogen $\left(10^{-4} M_{\odot}\right.$ to $\left.10^{-15} M_{\odot}\right)$, thus even the DB stars retain a small amount of hydrogen. The upper limit of the amount of hydrogen, $10^{-4} M_{\odot}$, corresponds to the limit where hydrogen burning stops. White dwarfs with only small amount of hydrogen are potentially regarded as DO/DB stars, while those with sufficient hydrogen are regarded as DA white dwarfs (Fontaine \& Wesemael 1997). The strong gravity of a white dwarf gradually leads to a sorting of the chemical elements with mild thin zones of varying chemical composition, and the hydrogen in the atmosphere of DO/DB stars is lifted to their surface (Schatzman 1958). Fontaine \& Wesemael (1987) suggested that, by the time the stars cool down to around $T_{\text {eff }} \sim 45000 \mathrm{~K}$, helium and hydrogen are well separated. The helium layer is masked by the hydrogen layer and no helium is spectroscopically visible, and then the DB/DO white dwarfs disappear. At $T_{\text {eff }} \sim 30000 \mathrm{~K}$, the formation of a helium ionization zone beneath the hydrogen layer creates turbulence which mixes hydrogen back into the subsurface layers, and leads to the reappearance of DB stars. Stars with more than $10^{-13} M_{\odot}$ of hydrogen have too much hydrogen to be well mixed, and they remain DA stars.

\section{An alternative working hypothesis}

As for the diffusion process, the time required for the separation of hydrongen and helium may be dependent on the amount of hydrogen. Furthermore, the population of white dwarfs may be fed by sources other than the planetary nebulae nuclei, such as subdwarf $\mathrm{O}$ and $\mathrm{B}$ stars, which are thought to be the hydrogen deficient stars going directly towards the white dwarf cooling from the extreme blue end of the horizontal branch as they do not have enough hydrogen to evolve along the asymptotic giant branch. Taking account of these, one might wonder why the cooling stage at $T_{\text {eff }} \sim 45000 \mathrm{~K}$ is thought to be the specific one at which diffusion makes hydrogen and helium to separate clearly. It seems more reasonable to suppose that a mechanism intrinsic to the white dwarfs' atmospheres with $T_{\text {eff }} \sim 45000 \mathrm{~K}$ does work to change the surface composition. It should be reminded that the temperatures of the blue and the red edges of the DB gap coincide with the effective temperatures where the He II/III and the He I/II convection zones show up respectively. Hence convective mixing is suspected of the cause of appearance of DBs outside the DB gap (Shibahashi 2005).

For $T_{\text {eff }}>45000 \mathrm{~K}$ the second helium ionization zone (He II/III) is shallow and becomes convectively unstable. Then, if the amount of hydrogen is sufficiently small, turbulence may thoroughly mix the hydrogen in the atmosphere with helium, leading to an apparent helium star, while stars having too much hydrogen to mix completely remain DA white dwarfs. At the later cooling stage, for $45000 \mathrm{~K}>T_{\text {eff }}>30000 \mathrm{~K}$, the He II/III convection zone is too deep to mix hydrogen, and gravitational settling that separates hydrogen and helium clearly leads to a hydrogen surface envelope. Hence there may be no DB/DO white dwarfs in this temperature range. Gravitational settling is very efficient in white dwarfs and the time scale required for the chemical separation is short enough (Fontaine \& Michaud 1979, Paquette et al. 1986). As Fontaine \& Wesemael (1987) suggested, at $T_{\text {eff }} \sim 30000 \mathrm{~K}$, the first helium ionization (He I/II) zone creates turbulence near the surface which mixes hydrogen with helium, and leads the star again to be a DB white dwarf (stars having too much $\mathrm{H}$ to mix remain DAs). In this new scenario, the presence of helium white dwarfs for $T_{\text {eff }}>45000 \mathrm{~K}$ and $T_{\text {eff }}<30000 \mathrm{~K}$ is a 
consequence of turbulent mixing, and chemical separation due to gravitational settling in the convectively stable atmosphere is proposed to be the cause of the presence of the DB gap.

\section{Semiconvection}

If one adopts either the spectral evolution model described in $\S 2$ or the alternative new hypothesis described in $\S 3$, the atmosphere of white dwarfs in the DB gap must be convectively stable. This usually means that the temperature gradient is subadiabatic. The temperature gradient of the atmosphere gradually becomes steeper when the stars cool down more and the surface atmosphere will eventually become to be convectively unstable to mix hydrogen with helium. However, since the stars located near the red edge of the DB gap are at the transition phase from the radiative atmosphere to the convective one, they are expected to have a superadiabatic layer, which is nonetheless still convectively stable due to the $\mu$-gradient which is caused by chemical separation.

A zone of varying chemical composition having a superadiabatic temperature gradient which is convectively stablized by a $\mu$-gradient is called a semiconvective zone. It is known that a semiconvective zone appears in a star more massive than $10 M_{\odot}$ during the main sequence evolution. Though semiconvective zones are dynamically (convectively) stable, overstable convection occurs in a medium of varying molecular weight if the temperature gradient is superadiabatic. This was first pointed out by Kato (1966). A similar situation was studied by Veronis (1965) in the salinity convection.

Overstability is a problem of pulsational instability of nonradial g-modes, and it can be settled only by global stability of a whole star (Shibahashi \& Osaki 1976). It is instructive, however, to discuss the mechanism by using a local analysis. Let us regard the white dwarf envelope as a plane-parallel, gravitationally stratified layer in hydrostatic and radiative equilibrium. We treat the gas as an ideal gas and consider high overtone gravity waves whose wavelengths in the vertical direction are much shorter than the density and pressure scale heights. In this situation, the Boussinesq approximation is appropriate. If the temporal and spatial dependence of the Eulerian perturbation of an arbitrary quantity $f$ is taken as

$$
f^{\prime}(x, z, t) \propto \exp \left[i\left(k_{h} x+k_{z} z\right)+s t\right]
$$

where $x$ denotes the horizontal direction and the gravity is the $z$-direction, then the basic equations of oscillations lead, in the first approximation of small nonadiabaticity, to the dispersion relation

$$
s \simeq \frac{K k^{2}}{2 c_{p} p} \frac{g^{2}}{N^{2}}\left(\nabla-\nabla_{\mathrm{ad}}\right) \pm i \frac{k_{h}}{k} N,
$$

where $k^{2} \equiv k_{h}^{2}+k_{z}^{2}, K \equiv\left(4 a c T^{3}\right) /(3 \kappa \rho)$ is the radiative conductivity, $g$ denotes the gravitational acceleration, $p$ denotes the pressure, and $c_{p}$ denotes the specific heat per unit mass at constant pressure. Here, $N$ means the Brunt-Väisälä frequency given by

$$
N^{2}=g^{2} \frac{\rho}{p}\left[\left(\nabla_{\mathrm{ad}}-\nabla\right)+\nabla_{\mu}\right]
$$

where $\rho$ denotes the density, $\nabla_{\text {ad }} \equiv(\partial \ln T / \partial \ln p)_{S}, \nabla \equiv d \ln T / d \ln p$, and $\nabla_{\mu} \equiv$ $d \ln \mu / d \ln p$, where $\mu$ denotes the mean molecular weight, $T$ denotes the temperature, and $S$ means the entropy. The necessary and sufficient condition for stability is $\Re(s)<0$. Dynamical instability occurs if $N^{2}<0$, that is, if

$$
\left(\nabla-\nabla_{\mathrm{ad}}\right)-\nabla_{\mu}>0
$$


which is often called the Ledoux (1947) criterion for convective instability. In the dynamically stable case, the medium is stable against ordinary convection since $N^{2}>0$, but perturbations grow to become oscillatory with increasing amplitude if

$$
\nabla-\nabla_{\mathrm{ad}}>0
$$

because the real part of $s$ in (4.2) is positive. The physical cause of this overstability is that the radiative heat exchange brings about an asymmetry in the oscillatory motion in such a way that an oscillatory element overshoots its equilibrium position with an increasing velocity.

\section{Pulsation is predicted}

The possibility of overstability of the semiconvective zone in a white dwarf was discussed by Baglin (1971) for a star with $T_{\text {eff }} \simeq 13000 \mathrm{~K}$. When she first discussed the semiconvection in a white dwarf, the characteristics of eigenfunctions of g-modes were not taken into account properly. Later, it was shown that the acoustic and gravity waves which are responsible for nonradial oscillations are propagative in the radial direction only in restricted regions (see text, e.g., Unno et al. 1989).

Let us try to take into account of the characteristics of propagation of waves. To do so, we consider in this section the wave properties by assuming that the specific entropy is conserved during the oscillations. We also in this section take account of compressibility. The gravity waves propagate in the vertical direction only in the regions in which $\omega>N(z)$ and $\omega>k_{h} c(z)$, where $\omega$ is the frequency of the wave (corresponds to $i \omega=s$ in $(4.1))$ and $c(z)$ denotes the sound speed, and the vertical wave number $k_{z}$ is approximately given by (see text, e.g., Unno et al. 1989)

$$
k_{z}(z) \simeq \frac{1}{\omega c}\left(N^{2}-\omega^{2}\right)^{1 / 2}\left(k_{h}^{2} c^{2}-\omega^{2}\right)^{1 / 2} .
$$

To get a rough sketch, we approximate here the white dwarf atmosphere as a polytrope, $\rho(z)=\rho_{0} z^{n}$, where $z$ is the depth from the surface. From the hydrostatic condition, $p(z)=(n+1)^{-1} \rho_{0} z^{n+1}$, and then,

$$
N^{2}(z) \equiv g\left(\frac{d \ln \rho}{d z}-\frac{1}{\gamma} \frac{d \ln p}{d z}\right)=\frac{G M}{R^{3}} \frac{R}{z}\left(n-\frac{n+1}{\gamma}\right),
$$

where $M$ and $R$ denote the mass and the radius of the star, respectively, and $G$ is the gravitational constant. We treat the upper layer above the semiconvective zone related with the $\mathrm{HeI} / \mathrm{II}$ ionization zone as the pure hydrogen atmosphere, where hydrogen is fully ionized. There the mean molecular weight $\mu=1 / 2$. The layer just beneath the semiconvective zone is assumed to be the pure HeI atmosphere, where $\mu=4$. The hydrogen profile in the semiconvection zone is assumed to be linear. Then the BruntVäisälä frequency in the semiconvective zone $\left(z=z_{*}\right)$ is roughly given as $N^{2}\left(z_{*}\right) \simeq$ $G M R^{-3} d \ln \mu / d z \simeq G M R^{-2}(14 / 9 h)$, where $h$ is the thickness of the semiconvective zone. If we take the pressure scale height as $h$, then

$$
N^{2}\left(z_{*}\right) \simeq \frac{G M}{R^{3}} \frac{R}{z_{*}} \frac{14(n+1)}{9},
$$

while $N^{2}(z) \simeq G M R^{-3}(3 / 5)(R / z)$ above the semiconvective zone $\left(z<z_{*}\right)$, and $N^{2}(z) \simeq$ 0 just beneath the semiconvective zone as the temperature gradient is almost the adiabatic temperature gradient and the chemical composition is homogeneous there. 
If we extend our picture to the more realistic geometry of a spherical star, the horizontal wave number $k_{h}$ should be replaced with the spherical degree $\ell$ as $k_{h}(\ell)=\{\ell(\ell+1)\}^{1 / 2} / R$. Since the sound speed is given by $c=\gamma p / \rho$, where $\gamma \equiv(\partial \ln p / \partial \rho)_{S}, k_{h} c(z)$ is approximately given by

$$
k_{h}^{2}(\ell) c^{2} \simeq \frac{G M}{R^{3}} \frac{\gamma}{n+1} \ell^{2} \frac{z}{R}
$$

We should note that the helium ionization zone is very shallow, $z_{*} / R \simeq 10^{-3}$. Hence $N^{2}\left(z_{*}\right) \simeq 6 \times 10^{3} G M / R^{3}$ while $k_{h}^{2}(\ell) c^{2}\left(z_{*}\right) \simeq 10^{-3} \ell^{2} G M / R^{3}$ when $n \simeq 3$.

The favorable condition for overstability is realized when g-modes are well trapped in the superadiabatic $\mu$-gradient zone and the amplitude outside the $\mu$-gradient zone is very small so that damping by the outer layers is negligible. This condition is satisfied in the case of high frequency, high degree g-modes with $\ell \geqslant 1000$, since $k_{h}(\ell) c\left(z_{*}\right)>N\left(z_{*}\right)>\omega$ in the $\mu$-gradient zone and $k_{z}^{2}<0$ outside there. The horizontal wave number $k_{h}(\ell)$ of such high degree modes is large and this is also favorable for excitation, as seen in (4.2). Hence, the $\mu$-gradient zone under consideration is definitely pulsationally unstable for high degree g-modes. The eigenfrequency is estimated from the quantization condition,

$$
\int k_{z} d z=\left(n_{\mathrm{g}}+\varepsilon\right) \pi
$$

and the period is thus estimated to be of the order of $0.05 \mathrm{~s}$;

$$
\omega \simeq \frac{\ell}{n_{\mathrm{g}}} N\left(z_{*}\right) \frac{h}{R} \simeq(30 \sim 40) \times\left(\frac{G M}{R^{3}}\right)^{1 / 2},
$$

where $n_{\mathrm{g}}$ denotes the radial (vertical) order. The degree $\ell$ is so high that the pulsation is not detectable.

The low frequency, low degree g-modes with $\ell \simeq 1$ can also be well-trapped in the $\mu$ gradient zone, since $N\left(z_{*}\right) \gg k_{h}(\ell) c\left(z_{*}\right)>\omega$ in this case and $k_{z}^{2}<0$ outside there. From the quantization condition (5.5), the periods of such low degree modes are of the order of $(30 \sim 40) \times\left(G M / R^{3}\right)^{-1 / 2}$, which is of the order of $100 \mathrm{~s}$. For $\ell<\leqslant 4$, the pulsation is expected to be detectable. It should be reminded that low degree g-modes are efficiently excited in DAVs and DBVs as a consequence of wave trapping in the thin ionization zones and they are indeed detected. This implies that we can hopefully observationally confirm the present hypothesis by detecting such modes for white dwarfs near the red edge of the DB gap. Discovery of pulsation in them will open up a new class of pulsating white dwarfs to asteroseismic study.

\section{Discussion}

Either explanation given by Fontaine \& Wesemael (1987) or the new one given here suggests that some fraction of white dwarfs in the DB gap, which are apparently classified as DA white dwarfs, have a helium layer beneath the surface hydrogen layer and return to become DBs again with cooling down as a consequence of turbulent mixing due to helium ionization. One of the issues at this stage is whether convective overshooting really occurs to mix the helium layer and the upper hydrogen layer. Careful treatment is required.

Another issue is the lifetime of the semiconvection phase. If the lifetime is shorter than the growth time of pulsation, the pulsation amplitude will not be able to become large enough to be detected. Also the chance of finding the stars near the red edge of the DB gap becomes lower. The growth time is of the order of the local thermal time scale of 
the semiconvective zone. It is shorter for the smaller wave number. This is favorite for low degree g-modes. A careful comparison of the growth time of the instability and the cooling time of white dwarfs near the red edge of the DB gap is necessary.

\section{Acknowledgements}

As the author had to cancel unexpectedly just before the conference his attendance to the symposium because of his wife's serious sickness, the oral presentation of this paper was kindly given on his behalf by Prof. Michael Thompson. The author expresses his sincere thanks to Prof. Thompson, as well as to the organizers of the symposium who kindly gave special consideration.

\section{References}

Baglin, A. 1971, in: W.J. Luyten (ed.), White Dwarfs, IAU Symp. 42 (Dordrecht: Reidel), p.136 Bergeron, P., Ruiz, M.T. \& Leggett, S.K. 1997, ApJS 108, 339

Fontaine, G. \& Michaud, G. 1979, ApJ 231, 826

Fontaine, G. \& Wesemael, F. 1987, in: A.G.D. Philip, D.S. Hayes \& J. Liebert (eds.), The Second Conference on Faint Blue Stars, IAU Colloq. 95 (Schenectady: Davis), p.319

Fontaine, G. \& Wesemael, F. 1997, in: J. Isern, M. Hernandez \& E. Garxía-Berro (eds.), White Dwarfs, Proc. of the 10th European Workshop on White Dwarfs, Astrophys. and Space Science Library (Dordrecht: Kluwer), vol. 214, p.173

Hansen, B.M.S. \& Liebert, J. 2003, ARAA 41, 465

Kato, S. 1966, PASJ 18, 374

Ledoux, P. 1947, ApJ 105, 305

Liebert, J. 1986, in: K. Hunger, D. Schönberner \& N.K. Rao (eds.), Hydrogen Deficient Stars and Related Objects, IAU Colloq. 87 (Dordrecht: Reidel), p.367

Liebert, J., Wesemael, F., Hansen, C.J., Fontaine, G., Shipman, H.L., Sion, E.M., Winget, D.E. \& Greenm R.F. 1986, ApJ 309, 241

Paquette, C., Pelletier, C., Fontaine, G. \& Michaud, G. 1986, ApJS 61, 197

Schatzman, E. 1958, White Dwarfs (Amsterdam: North-Holland)

Shibahashi, H. 2005, in: G. Alecian, O Richard \& S. Vauclair (eds.), Element Stratification in Stars: 40 Years of Atomic Diffusion, EAS Publ. Ser. (Les Ulis: EDP Science), vol. 17, p.143

Shibahashi, H. \& Osaki, Y. 1976, PASJ 28, 199

Sion, E.M. 1984, ApJ 282, 612

Unno, W., Osaki, Y., Ando, H., Saio, H. \& Shibahashi, H. 1989, Nonradial Oscillations of Stars, 2nd edition (Tokyo: Univ. of Tokyo Press)

Veronis, G. 1965, J. Marine Res. 23, 1

\section{Discussion}

KUPKA: Just a comment: M. Montgomery and I published a paper two years ago (Montgomery, M.H. \& Kupka, F. 2004, MNRAS 350, 267) on the importance of overshooting for DA and DB white dwarfs as found from using a non-local convection model. Overshooting might have to be taken into account in seismological or other kind of predictions for these variable stars and they also might be a means of probing models of overshooting and mixing.

SHIBAHASHI: I agree. 